the possible prohibition of performing sports, to the results of surgery as scars, a possible source of shame.

Parents of children with heart disease may experience higher stress levels than normal parental function and may feel very stressed about issues related to accountability and social integration.

Methods This research aims to investigate the quality of life in pediatric age both in terms of purely cardiological aspects (CardioPeds) and of general quality of life (PedsQL), also evaluating, starting from 12 years, the possible presence of depressive symptoms (PHQ-9) or anxious symptoms (GAD-7). These tests were administered both to the children and the parents, with the addition for these last ones of the compilation of the PSI, to investigate the parental distress.

The research allowed to divide the sample of 500 patients into 6 predominant pathologies, in order to compare the quality of life of children in different diseases and to understand if a specific pathology is associated or not with a lower quality of life.

Results Through an adequate statistical analysis it was found that the quality of life perceived by the subjects included in the study is significantly better than that perceived by the parents about the same children and adolescents.

Conclusions This study was one of the first to investigate the quality of life in congenital and/or acquired pediatric heart disease. However, the results obtained require further studies, in order to deepen what has emerged.

\section{GP35 STUDY OF THE STIFFNESS OF THE VASCULAR WALL IN CHILDREN FROM FAMILIES WITH A BURDENED HISTORY OF CARDIOVASCULAR DISEASES}

\begin{abstract}
1,2Evgeniia Slastnikova*, 'Dinara Sadykova, ${ }^{3}$ Irina Leontyeva, 'Liliya Galimova. 'Children's Republican Clinical Hospital of the Republic of Tatarstan Ministry of Health, KAZAN, Russian Federation; ${ }^{2}$ FSBEI HE Kazan SMU MOH Russia, Kazan, Russian Federation; ${ }^{3}$ Veltischev Research and Clinical Institute for Pediatrics of the Pirogov Russian National Research Medical University, Moscow, Russian Federation
\end{abstract}

10.1136/archdischild-2019-epa.101

Medical emergency center in Kazan conducted targeted screening to select patients with ischemic strokes, myocardial infarctions, angina pectories, widespread atherosclerosis of peripheral vessels, angiographically confirmed coronary atherosclerosis, ischemic heart disease and dislipidemia. 230 patients were selected (men $\leq 55$ years, women $\leq 60$ years). Their children, grandchildren (144 people) (Group 1) aged 5-17 years were examined. Control group of conditionally healthy children (112 people) was formed (Group 2), their family history was collected with the aim of excluding heredity burdened by CVD. A biochemical blood test was performed to determine the lipid profile; thus, a group with dyslipidemia (Group 1a) was selected among children with burdened family history, which included 76 people (53\%), lipidogram indicators (Group 1b) that were normal (68 people, 47\%). Rigidity indices of main vessels were determined: PTT (pulse wave propagation time), PWVao (pulse wave propagation speed in the aorta), AIx (augmentation index), (dP/dt) max ( $\max$ blood pressure rise rate). An ambulatory daily monitor, BPLab ${ }^{\circledR}$ software using Vasotens ${ }^{\circledR}$ technology was used.

Results In general, in Group 1, the rigidity indicators of the vascular wall were similar (average daily PTT $145.3 \pm 15.9$ ms, PWVao $6.7 \pm 0.9 \mathrm{~m} / \mathrm{s}$, AIx $4.9 \pm 8.1 \%$, (dP/dt) $\max$
$667.9 \pm 119.8 \mathrm{~mm} \mathrm{Hg} / \mathrm{s}$ ) with those of Group 2 (average daily PTT $140.5 \pm 15.2 \mathrm{~ms}$, PWVao $6.4 \pm 1.1 \mathrm{~m} / \mathrm{s}$, AIx 5.1 $\pm 8.2 \%,(\mathrm{dP} / \mathrm{dt}) \max 668.1 \pm 121.3 \mathrm{~mm} \mathrm{Hg} / \mathrm{s})$ and were statistically significant $(\mathrm{p}<0.05)$. However, it was found that if we consider non-maximum daily indicators, then in Group 1 they were significantly higher $(\mathrm{p}<0.05)$ ( $\max$ daily PTT $199.9 \pm 10.2 \mathrm{~ms}$, PWVao $16.3 \pm 1.4 \mathrm{~m} / \mathrm{s}$, AIx $35.3 \pm 3.4 \%$, $(\mathrm{dP} / \mathrm{dt}) \max 1098.1 \pm 120.5 \mathrm{~mm} \mathrm{Hg} / \mathrm{s})$ than in Group 2 (max daily PTT $181.7 \pm 9.6 \mathrm{~ms}$, PWVao $13.1 \pm 1.9 \mathrm{~m} / \mathrm{s}$, AIx $28.3 \pm 1.4 \%,(\mathrm{dP} / \mathrm{dt}) \max 1005.1 \pm 110.3 \mathrm{~mm} \mathrm{Hg} / \mathrm{s})$. Comparing the parameters between Group $1 \mathrm{a}$ and $1 \mathrm{~b}$, no statistically significant difference was found between the mean values, but statistically significant difference was found between the maximum values.

Conclusions Direct relationship has been revealed between the presence of a burdened family history of CVDs, changes in the lipid profile of parents, relatives of the 1 st and 2 nd lines, and changes in the rigidity of the wall of the great vessels in their children. The rigidity of the vascular wall was significantly higher in Group 1 than in Group 2, differed between children from Group 1a and Group 1b.

\section{GP36 FEEDING DIFICULTIES IN CHILDREN WHO UNDERWENT CARDIAC SURGERY FOR COMPLEX CONGENITAL HEART DISEASE IN KOSOVO}

1,2 Ramush Beiiqi* ${ }^{1}$ Ragip Ratkoceri, ${ }^{3}$ Hana Beijqi, ${ }^{3}$ Rinor Bejiqi, ${ }^{1}$ Arlinda Maloku, ${ }^{1}$ Aferdita Mustafa. ${ }^{1}$ Pediatric Clinic, University Clinical Center of Kosovo, Prishtina, Albania; ${ }^{2}$ Medical School, University Of Gjakova, i, Gjakova, Kosovo, Albania; ${ }^{3}$ Main Center of Family Medicine, Prishtina, Kosaovo, Prishtina, Albania

\subsection{6/archdischild-2019-epa.102}

Introduction A feeding disorder in infancy and during childhood is a complex condition involving different symptoms such as food refusal and faddiness, both leading to a decreased food intake. It often results from abnormal feeding development. Also, adequate nutrition is crucial and challenge in children after surgery for congenital heart disease. There is a worldwide reason for attention to lesion or specific feeding problems, supplementation of trace elements and minerals, and an organized approach to pace, timing, and type of feeding are beneficial.

Aim Of presentation is assessing the prevalence and predictors factors of feedings difficulties in children who underwent cardiac open heart surgery in neonatal period and infancy. We address selected nutritional and caloric requirements for children after cardiac surgery and explore nutritional interdependence with other system functions.

Method This was a retrospective study in a tertiary referral hospital, and prior approval from the institutional ethics committee was obtained. Information for 78 children (42 male and 36 female) was taken from patients charts. The presence of feeding difficulties or disorders was assessed by a questionnaire when the child was 3 years old. As a feeding disorder was defined as an inadequate food intake for age, failure of thrive or for few cases need for tube feeding. Data were analysed with descriptive statistics and logistic regression.

Results From cohort of analysed children feeding problems occurred in $23 \%$. At the time of study, refusal to eat or poor appetite was reported as a significant problem in 19 children and subnormal height and/or weight were recorded in 11 children. Early neonatal intervention and reoperation were identified as a risk factors for latter feeding difficulties 
or inadequate intake. Children with feeding problems also tended to eat less than children without feeding problems. There was a trend towards more feeding problems in patients with chromosomal abnormalities or other associated anomalies.

Conclusion Feeding disorder is often and frequent long-term sequel in children after neonatal or early infancy heart surgery. Patients with chromosomal and associated anomalies and those who underwent multiple cardiac surgeries are at risk of developing feeding difficulties and later feeding disorders. These patients need to be selected for preventive strategies and nutritional intervention should be offered in order to increase the caloric intake of the child and to develop a sound feeding relationship in the family.

\section{GP37 ECHOCARDIOGRAPHIC FOLLOW-UP OF CHILDREN WITH SUPRAVALVULAR AORTIC STENOSIS}

${ }^{1}$ Betül Çınar*, ${ }^{2}$ Sezen Ugan Atik, ${ }^{2}$ Ayşe Güler Eroğlu, ${ }^{2}$ Levent Saltık. ${ }^{1}$ Istanbul University Cerrahpaşa Medical Faculty, Department of Pediatrics, istanbul, Turkey; ${ }^{2}$ Istanbul University Cerrahpaşa Medical Faculty, Department of Pediatric Cardiology, istanbul, Turkey

\subsection{6/archdischild-2019-epa.103}

Introduction Supravalvular aortic stenosis (SVAS) is the congenital stenosis of the ascending aorta, which may be discrete or diffuse. It can be seen in children with no other underlying disease, especially in syndromic cases such as Williams syndrome. Supravalvular aortic stenosis is also seen as an autosomal dominant type of familial and sporadic cases. In this study, long-term and postoperative follow-up results of patients who were followed up between 1990 and 2017 were presented.

Materials and methods The study included 31 patients who were evaluated and followed with echocardiography at our institution between 1990 and 2017 with SVAS.

Results Twelve of the cases were female (39\%). Twenty patients were diagnosed with Williams syndrome. Thirty patients remained after exclusion of one case who was lost during cardiac catheterization, were followed for an average of $6.7 \pm 5.8$ years. The severity of SVAS detected in the first echocardiographic examination was very mild in 6 cases (19\%), mild in 8 cases (26\%), moderate in 5 cases $(16 \%)$ and severe in 12 cases (39\%). Six patients with severe SVAS were operated after the first echocardiographic examination. Right ventricular outflow tract stenosis was detected in the first examination in 6 cases and appeared in the third year of follow-up in one case. The mean right ventricular outflow tract obstruction gradient was $46.4 \pm$ $18.5 \mathrm{mmHg}$ on Doppler examination. According to the recent echocardiographic examination, the distribution of the degree of SVAS; Six patients $(19.3 \%)$ were very mild, 5 (16\%) were mild, 6 (19\%) were moderate, and $12(38.7 \%)$ were severe. SVAS progressed from very mild to mild in one patient, mild to moderate in 3 patients and moderate to severe in 3 patients. On the other hand, in 2 cases with severe SVAS were decreased during follow-up. Right ventricular outflow tract stenosis was lost in four cases and there was no change in three cases. Peripheral pulmonary stenosis (PPS) was present in 14 patients and PPS progressed in 2 patients, remained same in 7 cases and regressed in 5 cases. In the first examination, 21 of the cases had no aortic regurgitation; one patient had very mild, 5 had mild, and 5 had moderate aortic regurgitation gradually. Fourteen patients did not develop aortic regurgitation up to last examination.

Conclusion SVAS is a rare cause of left ventricular outflow tract obstruction requiring echocardiographic close follow-up

\section{GP38 SPECKLE TRACKING AS A MODERN ECHOCARDIOGRAPHIC TECHNIQUE IN ASSESSING CARDIAC FUNCTION IN CHILDREN}

${ }^{1,2}$ Andrada Mara Ardelean*${ }^{*},{ }^{1}$ Ruxandra Steflea, ${ }^{1,2}$ Ramona Stroescu, ${ }^{1,2}$ Raluca Isac,
${ }^{1,2}$ Cristina Olariu, ${ }^{1,2}$ Mihai Gafencu, ${ }^{1,2}$ Anca Popoiu, ${ }^{1,2}$ Gabriela Doros. ${ }^{1}$ Victor Babes
University of Medicine and Pharmacy, Timisoara, Romania; ${ }^{2}$ Illrd Peditric Clinic, Louis
Turcanu Emergency Hospital for Children, Timisoara, Romania

10.1136/archdischild-2019-epa.104

Introduction Speckle tracking is a new method of assessing cardiac function in adults, but used only at a research level in children. The aim is to implement this method in children with congenital and acquired cardiac pathology and oncologic disease as a new method of detecting cardiac function deterioration.

Methods and material We selected patients with congenital or acquired heard disease and oncologic pathology then performed echocardiography, cardiac biomarkers and speckle tracking and compared the results.

Results Currently we have 31 patients out of which $55 \%$ had a cardiac pathology, 26\% had an oncological disease and 19\% were collagenases or vasculitides. However, the study is ongoing and we hope to achieve a larger patient pool.

When considering the patients with congenital heart disease $47 \%$ had modified GLS(global longitudinal strain) and cardiac biomarkers, but a normal EF(ejection fraction). Aortic coarctation, aortic regurgitation, complex cyanogenic malformations, ventricular septal defect, complete AV block, hypertrophic cardiomyopathy and dilated cardiomyopathy were the maladies with altered GLS but with normal ejection fraction.

In the case of the complete AV block, she had a new dual chamber pacemaker installed, after which she developed symptoms of cardiac failure, GLS was under normal values, EF was $54 \%$ and had a septal dyssynchrony, however after modifying the device's parameters the GLS was normal and there was no septal dyssynchrony.

Patients with chemotherapy frequently develop cardiac toxicity. $44 \%$ of patients were diagnosed with cardiotoxicity with the help of speckle tracking and cardiac biomarkers.

One patient with Rhabdomyosarcoma with an initial normal GLS, chemotherapy was initiated, after which he accused palpitations. The GLS was at the lower normal limit, thus confirming cardiotoxicity due to Doxorubicin.

One patient with LES was monitored by speckle tracking. Initial normal GLS, was altered during a disease flare after which pericarditis developed.

We had two patients with Kawasaki disease complicated with severe coronary aneurysms which were followed-up by speckle tracking.

Conclusions Speckle tracking can be used as a fast and safe method to determine cardiac function in children, being well correlated with cardiac biomarkers, even when EF is normal. 